\title{
Limited Value of Staging Squamous Cell Carcinoma of the Anal Margin and Canal Using the Sentinel Lymph Node Procedure: A Prospective Study with Long-Term Follow-Up
}

\author{
Johannes S. de Jong, MD, PhD ${ }^{1}$, Jannet C. Beukema, $\mathbf{M D}^{2}$, Gooitzen M. van Dam, Md, PhD ${ }^{1}$, Riemer Slart, MD, \\ $\mathbf{P h D}^{3}$, Clara Lemstra ${ }^{3}$, and Theo Wiggers, $\mathbf{M d}, \mathbf{P h D}^{1}$ \\ ${ }^{1}$ Department of Surgery, Division of Surgical Oncology, University Medical Center Groningen, University of Groningen, \\ Groningen, The Netherlands; ${ }^{2}$ Department of Radiotherapy, University Medical Center Groningen, University of \\ Groningen, Groningen, The Netherlands; ${ }^{3}$ Department of Nuclear Medicine and Molecular Imaging, University Medical \\ Center Groningen, University of Groningen, Groningen, The Netherlands
}

\begin{abstract}
Background. Selection of patients with anal cancer for groin irradiation is based on tumor size, palpation, ultrasound, and fine needle cytology. Current staging of anal cancer may result in undertreatment in small tumors and overtreatment of large tumors. This study reports the feasibility of the sentinel lymph node biopsy (SLNB) in patients with anal cancer and whether this improves the selection for inguinal radiotherapy.

Methods. A total of 50 patients with squamous anal cancer were evaluated prospectively. Patients without a SLNB $(n=29)$ received irradiation of the inguinal lymph nodes based on lymph node status, tumor size, and location of the primary tumor. Inguinal irradiation treatment in patients with a SLNB was based on the presence of metastases in the SLN.

Results. SLNs were found in all 21 patients who underwent a SLNB. There were 5 patients $(24 \%)$ who had complications after SLNB and 7 patients (33\%) who had a positive SLN and received inguinal irradiation. However, 2 patients with a tumor-free SLN and no inguinal irradiation developed lymph node metastases after 12 and 24 months, respectively.

Conclusions. We conclude that SLNB in anal cancer is technically feasible. SLNB can identify those patients who
\end{abstract}

(C) The Author(s) 2010. This article is published with open access

at Springerlink.com

First Received: 8 July 2009;

Published Online: 14 April 2010

J. S. de Jong, MD, PhD

e-mail: j.s.dejong@chello.nl would benefit from refrain of inguinal irradiation treatment and thereby reducing the incidence of unnecessary inguinal radiotherapy. However, because of the occurrence of inguinal lymph node metastases after a tumor-negative SLNB, introduction of this procedure as standard of care in all patients with anal carcinoma should be done with caution to avoid undertreatment of patient who otherwise would benefit from inguinal radiotherapy.

The incidence of anal carcinoma is 4-7 per 1 million with a peak incidence between 58 and 64 years of age. Anal carcinoma is associated with chronic anorectal disorders, immunosuppression, and with the presence of Human Papilloma Virus. Of all patients presenting with anal carcinoma, $36 \%$ have T3-T4 tumors. ${ }^{1}$ Synchronous inguinofemoral metastasis are observed in $11 \%$ of all patients presenting with an anal carcinoma and in $10 \%$ of those treated with curative intent. ${ }^{1}$ The presence of lymph node metastasis in anal cancer is associated with a poor prognosis. Because surgery is reserved for persistent and recurrent disease, and removal of the inguinofemoral lymph nodes is not initially performed, there is little data on the histologic nodal status of patients that present with anal carcinoma. The current standard treatment for synchronous inguinal lymph node metastases is chemoirradiation of the inguinofemoral lymph node basins, and the occurrence of locoregional metastases can be controlled in $90 \%$ of patients. ${ }^{2}$ This leads to an important finding that early detection of inguinal lymph node metastases is therefore clinically relevant in anal carcinoma.

Assessing the lymph node involvement by clinical examination alone in the inguinofemoral region solely by 
palpation is disappointing since $44 \%$ of lymph node metastases measure $<5 \mathrm{~mm}$ in diameter. ${ }^{3}$ Ultrasound (US) is significantly more reliable compared with palpation with a reported sensitivity of $36 \%$ to $87 \%$ and a specificity ranging from $56 \%$ to $89 \% .{ }^{4}$ The addition of fine-needle aspiration (FNA) cytology may increase the detection rate of lymph node metastases up to $65 \% .^{5}$ However, considering the small size of the inguinal metastasis, even when all modalities are combined (i.e., clinical examination, ultrasound, computerized tomography [CT] scanning, MRI, FNA cytology), not all cases of nodal involvement will be detected. Clearly, there is a need for early and accurate detection of lymph node involvement that could select those patients who could benefit the most from inguinal radiotherapy for locoregional control. This could reduce the morbidity of unnecessary inguinal radiotherapy in these patients without lymph node involvement. A possible technique to solve this problem is the sentinel lymph node biopsy (SLNB). Sentinel lymph node biopsy has been used the improve the detection rate of lymph node metastases in patient with cutaneous squamous cell carcinoma in the anogenital region as recently reviewed by Ross et al. ${ }^{6}$ The detection rate of the sentinel lymph node (SLN) was $97 \%$ with $24 \%$ of the SLNBs being positive for tumor cells. The false-negative rate, as determined by subsequent lymphadenectomy irrespective of SLN status, appeared to be $4 \% .^{6}$ However, in patients with anal cancer there is very limited evidence of the applicability of SLNB. Most studies lack sufficient follow-up and do not include their clinical decision making on the basis of the SLNB status. As a consequence, this would imply irradiation of the groin in every positive SLNB, irrespective of tumor size and, consequently, for patients with a negative SLNB no irradiation on the groin.

This study describes the technical feasibility of the SLNB technique in patients with squamous anal carcinoma and the effect of inguinal irradiation treatment based on the SLNB status with sufficient follow-up.

\section{PATIENTS AND METHODS}

\section{Patient Group}

Between 2001 and 2008, 50 patients (27 women and 23 men, mean age 60 years, range 34-88 years) with histopathologically proven squamous carcinoma of the anal canal and anal margin were prospectively enrolled in the study after informed consent. The inguinal region was staged with palpation and US followed by US-guided cytology in case of suspected lymph nodes. Patients with positive inguinal lymph nodes on cytology were not eligible for the sentinel lymph node protocol. Patients were staged for distant metastases with ultrasonography of the liver and chest X-ray or a CT scan of the abdomen and thorax. Patients with evidence of distant metastasis were also excluded from the study. Tumor size was determined by endoscopy, endosonography, or MRI according to the World Health Organization (WHO) recommendations for anal cancer. ${ }^{7}$ Patients with T4 tumors or without written informed consent were excluded from the sentinel lymph node protocol. Of the 50 patients, 21 were eligible for a SLNB. The remaining 29 patients were included in the follow-up for evaluation of clinical outcome after standard treatment. Figure 1 summarizes the patient selection for SLNB.

\section{Lymphatic Mapping and Sentinel Lymph Node Biopsy}

One day prior to surgery, $0.5 \mathrm{~mL} 100 \mathrm{mBq}^{99 \mathrm{~m}}$ Tc-labeled nanocolloid (Nanocoll: Amersham Cygne, Eindhoven, The Netherlands) was injected intradermal in 3-4 locations around the primary tumor. For tumors in the anal canal, the ${ }^{99 \mathrm{~m}} \mathrm{Tc}$-nanocolloid was injected in 4 locations around the anus. For imaging purposes, a single-headed gamma camera (Diacam, Siemens, Hoffmann Estates, IL) was used equipped with low-energy all-purpose collimator. The energy window was centered on the ${ }^{99 \mathrm{~m}} \mathrm{Tc}$-photopeak of $140 \mathrm{keV}$, using a $15 \%$ window. Zoom factor was 1 , image matrix $128 \times 128$ pixels for dynamic, and $256 \times 256$ pixels for static acquisition. Anterior images of the pelvic and inguinofemoral region were obtained. Within $5 \mathrm{~min}$ after injection, dynamic imaging was started with 30-s frames during $30 \mathrm{~min}$. An anterior and lateral static image (bilateral in case of double-sided lymph nodes) was obtained 2.5-3 h postinjection. In order to facilitate interpretation, transmission scanning was performed simultaneously using the $120 \mathrm{keV}$ gamma rays of a ${ }^{57} \mathrm{Co}$ flood source. The firstappearing persistent focal accumulation was considered to be a SLN. The site of the SLN was marked with a waterproof pencil on the overlying skin. On the day of surgery and following induction of anesthesia, approximately $0.5-2.0 \mathrm{ml}$ Patent Blue (Laboratoire Guerbet, Aulney-Sous-Bois, France) was injected subcutaneously in the same locations as the nanocolloid, $\sim 5-10 \mathrm{~min}$ prior to the surgical procedure. The SLNB was guided by a handheld gamma ray detection probe (Neoprobe, Johnson \& Johnson Medical, Hamburg, Germany) and by dissection of blue-stained lymph vessels. When identification of the SLN was not successful because of low radioactivity during the operative procedure, the use of Patent Blue was relied on. The blue nodes found in this manner were checked for radioactivity after excision. After removal of the SLN(s), the biopsy bed was re-examined for radioactivity. Lymph nodes that appeared to be affected by palpation, even if they were not radioactive or blue, and blue 
FIG. 1 Selection of patients for SLNB and inguinal radiotherapy and the incidence of inguinal lymph node recurrence after completion of treatment

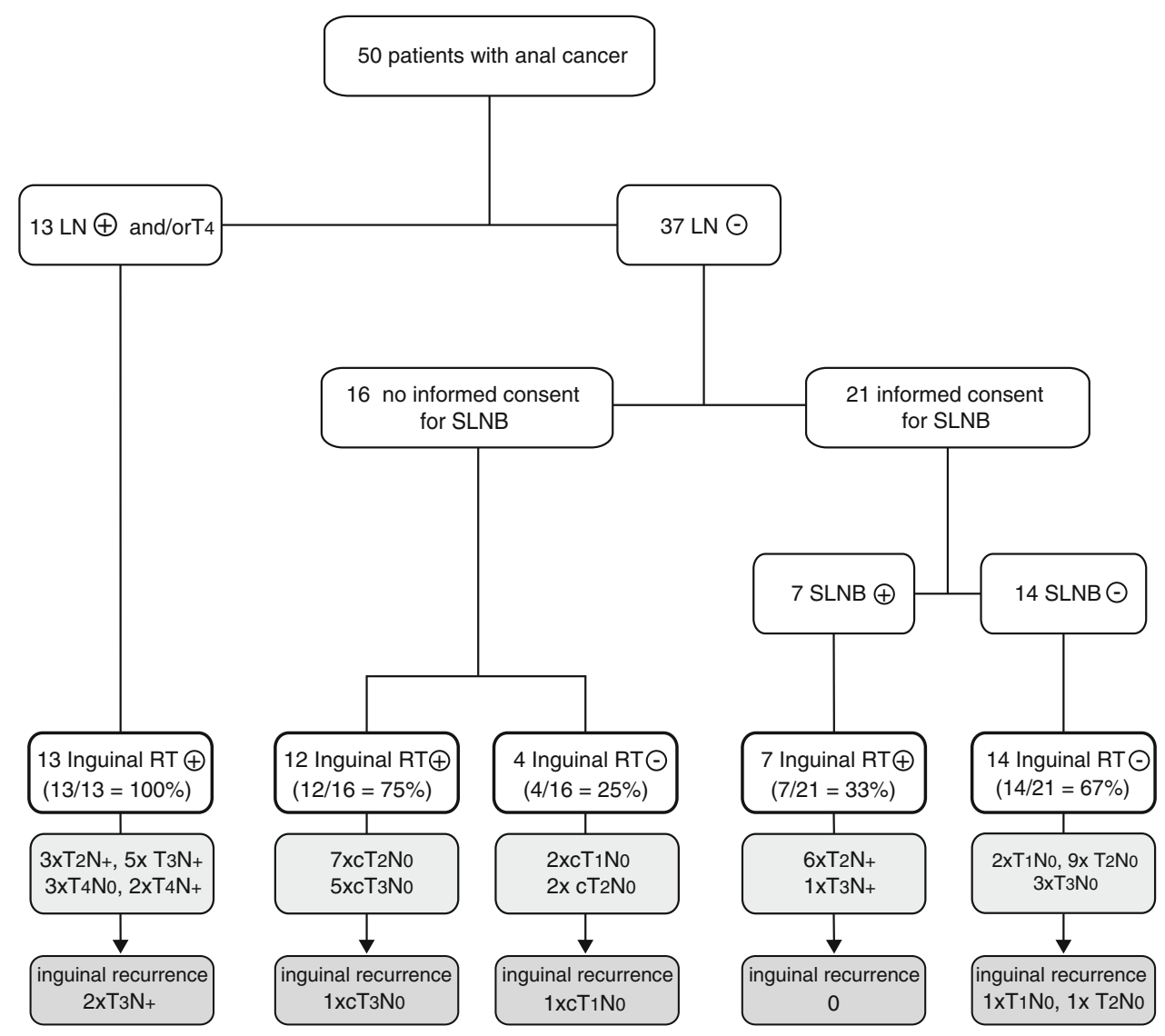

nodes that were not radioactive were removed as well and evaluated for the presence of tumor cells.

\section{Histopathological Examination}

Lymph nodes $<0.5 \mathrm{~cm}$ in diameter were included in whole, those $\geq 0.5$ and $<1 \mathrm{~cm}$ were cut in half and imbedded completely, and lymph nodes $\geq 1 \mathrm{~cm}$ were lamellated and processed to paraffin blocks for hematoxylin and eosin (H\&E) staining. SLNs that were negative on H\&E staining were stained by immunohistochemistry (cytokeratin antibody $\mathrm{AE} 1 / 3$ ) at $250 \mu \mathrm{m}$ intervals sections at 3 levels.

\section{Treatment}

Patients received irradiation of the primary tumor and locoregional lymph nodes (single dose $1.8 \mathrm{~Gy}, 25$ fractions, boost primary tumor and involved lymph nodes; single dose $1.8 \mathrm{~Gy}, 8$ fractions) combined with chemotherapy using 5-fluorouracil/mitomycine-C or capecitabine/ mitomycine-C. Patients with small tumors T1 $(\leq 2 \mathrm{~cm})$ and no nodal involvement did not receive irradiation of the inguinal lymph nodes. Node-negative T2 tumors with growth confined to the anal canal were inspected by the radiotherapist and were considered for radiotherapy of the primary tumor alone.

Irradiation treatment of the patients in the SLNB group was based on the inguinal lymph node status irrespective of their T-stage. If the SLNB was positive, the radiation field was extended to the inguinal lymph nodes. In cases of negative SLNB, the patient received standard treatment of the primary tumor if large enough without irradiation of the inguinal lymph nodes.

Radiation without chemotherapy ( $n=14$ ) was given in selected patients with stage $\mathrm{T} 1$ or contraindications for chemotherapy. Follow-up for all patients was every 3 months with a median follow-up period of 31 months (range 20-90 months). Follow-up consisted of visual inspection and palpation of the primary tumor side and the groin region.

\section{RESULTS}

\section{Patient Characteristics and TNM Stage}

Of the 50 patients enrolled, 21 underwent the SLNB procedure. Of the patients in the SLNB group, the majority had stage T2 primary tumors ( 2 were T1, 15 were T2, 4 were T3) (Fig. 1). In the patients with a SLNB, US of the 
groin showed suspected lymph nodes in 4 patients (19\%). US-guided fine-needle cytology of these lymph nodes was overall negative. However, 2 of these 4 patients with negative fine needle cytology had a positive SLNB and received irradiation of the inguinal region.

The 29 patients without a SLNB had mainly T2 and T3 tumors ( 2 were T1, 12 were T2, 10 were T3, 5 were T4). Of these patients, 11 had lymph node involvement detected by palpation or US followed by US-guided cytology (Fig. 1).

\section{Sentinel Lymph Node Detection}

All 21 patients showed 1 or more SLNs on the preoperative lymphoscintigram. Of the 21,7 patients (33\%) had a unilateral SLN and $14(67 \%)$ showed bilateral SLNs on the lymphoscintigram. A total of 58 SLNs were seen on the lymphoscintigram. Of the 58 SLNs, 43 (75\%) of the lymph nodes seen on the lymphoscintigram were detected with Patent Blue staining; 53 of the 58 lymph nodes identified on the lymphoscintigram (91\%) were detected with the handheld gamma probe.

Of 21 patients, 7 (33\%) had tumor metastases in the SLNB. Also, 5 of 21 patients (23\%) had complications after the SLNB; 2 patients experienced wound infection, 2 had seroma, and 1 patient developed lymphedema of the leg. Of these 5 patients, 4 had a positive SLN and had to undergo irradiation of the groin. Radiotherapy of the groin had to be postponed for several weeks in all cases of wound infection.

\section{Treatment and Tumor Response}

The standard treatment of patients with anal cancer consisted of a combination of irradiation and chemotherapy in 37 patients $(74 \%$ ) (21 without a SLNB and 16 with a SLNB procedure). Of the patients who received chemotherapy, $51 \%$ received a combination of 5-fluorouracil and mitomycine-C and $49 \%$ a combination of capecitabine and mitomycine-C. Chemotherapy treatment was stopped for 3 patients after 1 dose because of side effects. All patients received irradiation therapy of the primary tumor. A total of 45 of the patients $(90 \%)$ had a complete response (CR; defined as no residual tumor found by visual inspection and palpation) after treatment of the primary tumor. Of the 5 patients (10\%) (all without a SLNB) with a partial response (PR) 4 developed distant metastases within 1 year of initial treatment and therefore refrained from salvage surgery. The other patient with a partial response was treated with an abdominal perineal resection.

Of the patients without a SLNB, all 13 patients with lymph node metastasis or a $\mathrm{T} 4$ tumor received irradiation of the inguinal lymph nodes. Of clinical node-negative patients without informed consent for a SLNB, 75\% (12 of 16) received irradiation of the inguinal lymph nodes based on the tumor size and tumor localization. In the group of patients with a SLNB, 33\% (7 of 21) of patients were treated with extension of the irradiation field to the inguinofemoral region based on a tumor positive SLNB (Fig. 1). Complications after irradiation of the inguinofemoral region consisted mainly of moderated or severe epidermolysis of the skin. Of the 32 patients who received irradiation of the inguinofemoral region, $15(47 \%)$ had grade 1 and 17 (53\%) developed grade 2 complications.

\section{Outcomes and Survival}

Of the patients with a SLNB, 2 of 14 (14\%) developed inguinal lymph node metastases despite a negative SLNB based on histopathology. The first patient was an 81-year-old man with a left lateral T1 tumor of $1 \mathrm{~cm}$ and $1 \mathrm{SLN}$ on the lymphoscintigram. The SLNB showed a blue and radioactive SLN on the right side without tumor cells at histopathological examination. As a consequence, the patient received irradiation of the primary tumor without additional chemotherapy and showed a complete response. The second patient was a 67-year-old man with a left lateral $\mathrm{T} 2$ tumor of $4 \mathrm{~cm}$. Clinical examination did not reveal suspected lymph nodes. On CT of the abdomen there were suspected deep iliac lymph nodes, but CT-guided fine-needle aspiration was negative for tumor cells. The lymphoscintigram showed 3 SLNs on the right side and one SLN on the left side. The SLNB itself showed 3 blue + radioactive SLNs on the right side that were tumor negative at histopathology. No blue or radioactive SLN could be detected on the left side. The patient received irradiation of the primary tumor with capecitabine/Mitomycine-C chemotherapy and showed a complete response. Both patients had recurrence in the bilateral inguinal lymph nodes after 12 and 24 months, respectively. These 2 patients were treated by superficial inguinal lymph node dissection and irradiation of the inguinofemoral region. The last patient developed a local recurrence of the primary tumor and underwent an abdominal perineal resection. Both patients died after 23 and 26 months follow-up, respectively.

In the group of patients without a SLNB, 1 of 29 patients (3\%) had an inguinal lymph node recurrence (Fig. 1). This patient had a cT1N0 tumor and was treated with irradiation of the primary tumor with a CR. This patient died at the end of study period of distant metastasis. After a median follow-up of 31 months the tumor-related 5 year survival was $76 \%$.

\section{Alteration of Treatment by SLNB}

The guidelines for treatment of anal carcinoma developed by the Dutch group for gastrointestinal tumors (www. oncoline.nl) states that irradiation of the inguinal lymph 
nodes is optional for T2 and T3 tumors and mandatory in T4 tumors. In our study design, SLNB provided alteration of treatment in at least 11 of the 21 patients: 6 patients with large $\mathrm{T} 2$ tumors $(>3 \mathrm{~cm})$ and 3 patients with $\mathrm{T} 3$ tumors revealed tumor-free inguinal SLN and would have received unnecessary irradiation under the standard treatment regime. There were 2 patients with small T2 tumors not invading the anal canal upstaged to lymph node positive patients based on the SLNB, and they received inguinal radiotherapy.

\section{DISCUSSION}

Treatment of patients with anal carcinoma and no clinical evidence of lymph node involvement is controversial. In a series of 153 patients treated with extended irradiation, inguinal recurrences were observed in only 4 patients $(3 \%){ }^{8}$ Prophylactic radiotherapy to inguinal fields has led to a decrease of $15 \%-25 \%$ to approximately $3 \%-4 \%$ in the risk of development of metachronous inguinal metastases. $^{9-12}$ However, in 2 series with a total of 490 patients treated with chemoradiation that spared the inguinal lymph nodes if not macroscopically involved, only $7.6 \%$ developed metachronous inguinal metastases. ${ }^{1,13}$

In this study we showed that SLNs could be detected in all patients with anal carcinoma who were eligible for SLNB. We used a combined ${ }^{99 \mathrm{~m}}$ Tc-labeled nanocolloid and Patent Blue technique to improve the yield of the SLNB technique with the lowest false negative rate. ${ }^{14,15}$ Currently, 8 studies with a total of 143 patients have been published that describe the feasibility of SLNB in patients with squamous carcinoma of the anal carcinoma. ${ }^{16-23}$ These studies showed a high SLN detection rate of $96.5 \%$ (138 of 143). The SLN could not be identified in all cases if ${ }^{99 m}$ Tc-nanocolloid was used as the only tracer for the SLN. Table 1 summarizes the results these 8 studies.

In our study, 2 patients showed inguinal lymph node metastases after a tumor negative SLNB. Since the lymph node metastases both were present within 2 years (12 and 24 months), we could consider these metastases as an initial false-negative SLN. Because most previous studies lack a long-term follow-up or alteration of the inguinal irradiation therapy based on the SLNB, the incidence of a false-negative SLN is not known. The only study with treatment alteration based on the SLNB was the recent study by Gretschel et al. ${ }^{23}$ In that study, 1 patient with a T1 tumor showed inguinal lymph node recurrence after a tumor-negative SLNB.

To improve the accuracy of the SLNB procedure, lymph node staging before the SLNB should be optimal. Ultrasound in combination with FNA of the lymph node basins has been used in melanoma, breast, and penile cancer to optimize the lymph node staging and reduce the number of unnecessary SLN procedures by 5\%-11\%..$^{24-27}$ In our study, 1 of the patients with lymph node recurrence after a tumor-negative SLNB showed suspected deep inguinal lymph nodes on the ultrasound but a tumor-negative FNA cytology. Of the 3 other patients with suspected lymph nodes on the ultrasound and tumor-negative FNA cytology, 2 had a tumor-positive SLNB. One could therefore argue that in order to improve the accuracy of the SLNB, suspected lymph node on ultrasonography should be treated as tumor-positive lymph nodes.

Irradiation of the inguinofemoral region can lead to serious complications with acute and late toxicity. Acute morbidity includes epidermolysis with ulceration and superinfection of the skin, while late toxicity includes inguinal fibrosis, external genitalia edema, neurogenic bladder, lower limb lymph edema, osteonecrosis of the femoral head, artery stenosis, and soft tissue sarcomas. ${ }^{1,12}$ In our study, 9 patients with T2 and T3 tumors had an alteration of therapy bases on a negative SLNB and did not receive groin irradiation. Of the 32 patients who received radiotherapy of the inguinofemoral basins, the complications were mainly moderate to severe epidermolysis of the skin. Because the median follow-up was 31 months, we could not determine the exact incidence of late toxicity. However, of the 9 patients who received inguinal radiotherapy with a follow-up of 5 years there was no incidence of late irradiation toxicity.

TABLE 1 Studies of SLNB in squamous cell carcinoma of the anal margin and canal

\begin{tabular}{|c|c|c|c|c|c|c|c|c|}
\hline Author & $\begin{array}{l}\text { Year of } \\
\text { publication }\end{array}$ & $\begin{array}{l}\text { Number } \\
\text { of patients }\end{array}$ & $\begin{array}{l}\text { Dye vs } \\
\text { isotope }\end{array}$ & $\begin{array}{l}\text { SLN(+)/SLN } \\
\text { identified }\end{array}$ & $\begin{array}{l}\text { Number of cases of } \\
\text { SLN not identified }\end{array}$ & $\begin{array}{l}\text { Lymphadenectomy } \\
\text { after SLNB }\end{array}$ & $\begin{array}{l}\text { Mean follow- } \\
\text { up (months) }\end{array}$ & $\begin{array}{l}\text { Nodal metastasis } \\
\text { during follow-up }\end{array}$ \\
\hline Peley $^{16}$ & 2002 & 8 & Both & $2 / 8$ & $0 / 8$ & No & $5-35$ & $0 / 8$ \\
\hline Rabbit $^{17}$ & 2002 & 4 & Both & $0 / 4$ & $0 / 4$ & No & None & \\
\hline Perera $^{18}$ & 2003 & 12 & Both & $2 / 12$ & $0 / 12$ & No & None & \\
\hline Ulmer $^{19}$ & 2003 & 17 & Isotope & $5 / 13$ & $4 / 17$ & No & 10 & $1 / 16$ \\
\hline $\operatorname{Damin}^{20}$ & 2003 & 14 & Both & $1 / 14$ & $0 / 14$ & No & None & \\
\hline Bobin $^{21}$ & 2003 & 33 & Both & $7 / 33$ & $0 / 33$ & No & 18 & $0 / 18$ \\
\hline Mistrangelo $^{22}$ & 2008 & 35 & Isotope & $7 / 34$ & $1 / 35$ & No & 21 & $0 / 34$ \\
\hline Gretschel $^{23}$ & 2008 & 20 & Isotope & $6 / 20$ & $0 / 20$ & No & 27 & $1 / 20$ \\
\hline
\end{tabular}


Improvement of staging of anal carcinoma with the SLNB procedure is not without complications. In our study, $24 \%$ of patients with a SLNB had a postoperative complication to the groin after the SLNB procedure. Since 4 of these 5 patients had a metastasis in the SLN, radiotherapy of the groin had to be postponed because of inguinal wound complication. This is in line with the study of Mistrangelo et al., which found a postoperative complication rate of $57 \%$ including inguinal lymphorrhea, monolateral lymph edema of the lower limb, and lymphocele. ${ }^{22}$ In a recent review of SLNB in cutaneous squamous cell carcinoma by Ross and Schmults, adverse events were reported rarely. ${ }^{6}$ However, since inguinal lymph node metastases of anal carcinoma are treated with irradiation, surgical complications caused by SLNB can have a negative effect on the morbidity of inguinal radiotherapy.

Surgical series have shown that about half the inguinal lymph node with metastasis are less than $5 \mathrm{~mm}$ in size. ${ }^{3}$ Because these small lymph nodes are not detectable by palpation or CT imaging, other noninvasive staging modalities would be more appropriate in patients with small tumors who otherwise would not be treated with groin irradiation. In a recent study by Nguyen et al., 18FDG PET showed upstaging and alteration of radiotherapy planning in $18 \%$ of N0 patient with anal cancer. ${ }^{28}$

We conclude that SLNB in anal cancer following careful primary selection based on clinical examination, imaging techniques, and FNA of suspected nodes is technical feasible with a high detection rate of SLNs. SLNB can be used to upstage clinical node-negative patients with small tumors who would otherwise refrain from inguinal radiotherapy. Besides, SLNB can identify those patients who would benefit from refrain of inguinal irradiation treatment and thereby reduce the incidence of unnecessary inguinal radiotherapy. However, because of the occurrence of inguinal lymph node metastases after a tumor-negative SLNB, introduction of this procedure as standard of care in all patients with anal carcinoma should be done with caution to avoid undertreatment of patient who otherwise would benefit from inguinal radiotherapy.

OPEN ACCESS This article is distributed under the terms of the Creative Commons Attribution Noncommercial License which permits any noncommercial use, distribution, and reproduction in any medium, provided the original author(s) and source are credited.

\section{REFERENCES}

1. Gerard JP, Chapet O, Samiei F, Morignat E, Isaac S, Paulin C, et al. Management of inguinal lymph node metastases in patients with carcinoma of the anal canal. Cancer. 2001;92:77-84.

2. Fuchshuber PR, Rodriguez-Bigas M, Weber T, Petrelli NJ. Anal cancer and perianal epidermoid cancers. J Am Coll Surg. 1997; 185:494-505.
3. Wade DS, Herrera L, Castillo NB, Peterelli NJ. Metastases to the lymph nodes in epidermoid carcinoma of the anal canal studied by a clearing technique. Surg Gynecol Obstet. 1989;169:238-42.

4. Mäkelä PJ, Leminen A, Kääriäinen M, Lehtovirta P. Pretreatment sonographic evaluation of inguinal lymph nodes in patients with vulvar malignancy. J Ultrasound Med. 1993;5:255-8.

5. Bonnema J, Van Geel AN, Van Ooijen B, Mali SP, Tjiam SL, Henzen-Logmans SC, et al. Ultrasound-guided aspiration biopsy for detection of nonpalpable axillary node metastases in breast cancer patients: new diagnostic method. World J Surg. 1997;21: $270-4$.

6. Ross AS, Schmults CD. Sentinel lymph node biopsy in cutaneous squamous cell carcinoma: a systematic review of the English literature. Dermat Surg. 2006;32:1309-21.

7. Sobin LH, Wittekind C. TNM Classification of Malignant Tumors. New York: Wiley-Liss, 2002.

8. Salmon RJ, Fenton J, Asselain B et al. Treatment of epidermoid anal cancer. Am J Surg. 1984;147:43-8.

9. Boman BM, Moertel CG, O'Connell MJ, Scott M, Weiland LH, Beart RW, et al. Carcinoma of the anal canal: a clinical and pathological study of 188 cases. Cancer. 1984;54:114-25.

10. Roelofsen F, Bartelink H. Combined treatment of anal carcinoma. Oncologist. 1998;3:413-8.

11. Mitchell SE, Mendenhall WM, Zlotecki RA, Carroll RR. Squamous cell carcinoma of the anal canal. Int J Radiat Oncol Biol Phys. 2000;49:1007-13.

12. Meyerson RJ, Kong F, Birnbaum EH, Fleshman JW, Kodner IJ, Picus J, et al. Radiation therapy for epidermoid carcinoma of the anal canal, clinical and treatment factors associated with outcome. Radiotherapy Oncol. 2001;61:15-22.

13. Papillon J, Montbarbon JF. Epidermoid carcinoma of the anal canal: a series of 276 cases. Dis Colon Rectum. 1987;30: 324-33.

14. Kapteijn BAE, Nieweg OE, Liem IH, Mooi WJ, Balm AJ, Muller $\mathrm{SH}$, et al. Localizing the sentinel node in cutaneous melanoma: gamma probe detection versus blue dye. Ann Surg Oncol. 1997;4:156-60.

15. Pijpers R, Borgstein P, Meijer S, Hoekstra OS, Van Hattum LH, Teule GJJ. Sentinel node biopsy in melanoma patients: dynamic lymphoscintigraphy followed by intraoperative gamma probe and vital dye guidance. World J Surg. 1997;21:788-93.

16. Peley G, Farkas E, Sinkovics I, Kovacs T, Keresztes S, Orosz Z, et al. Inguinal sentinel lymph node biopsy for staging anal cancer. Scand J Surg. 2002;91:336-8.

17. Rabbitt P, Pathma-Nathan N, Collinson T, Hewett P, Rieger N. Sentinel lymph node biopsy for squamous cell carcinoma of the anal canal. ANZ J Surg. 2002;72:651-4.

18. Perera D, Pathma-Nathan N, Rabbitt P, Hewett P, Rieger N. Sentinel node biopsy for squamous cell carcinoma of the anus and anal margin. Dis Colon Rectum. 2003;46:1027-31.

19. Ulmer C, Bembenek A, Gretschel S, Markwardt J, Koswig S, Slisow $\mathrm{W}$, et al. Sentinel node biopsy in anal cancer-a promising strategy to individualize therapy. Onkologie. 2003;26:456-60.

20. Damin DC, Rosito MA, Gus P, Spiro BL, Amaral BB, Meurer L, et al. Sentinel lymph node procedure in patients with epidermoid carcinoma of the anal canal. Dis Colon Rectum. 2003;46:1032-7.

21. Bobin JY, Gérard JP, Chapet O, Romestaing P, Isaac S. Lymphatic mapping and inguinal sentinel lymph node biopsy in anal cancers to avoid prophylactic inguinal irradiation. Cancer Radiother. 2003;7:85s-90s.

22. Mistrangelo M, Bello M, Mobiglia A, Beltramo G, Cassoni P, Milanesi E, et al. Feasibility of the sentinel node biopsy in anal cancer. Q J Nucl Med Mol Imaging. 2009;53:3-84.

23. Gretschel S, Warnick P, Bembenek A, Dresel S, Koswig S, String A, et al. Lymphatic mapping and sentinel lymph node biopsy in 
epidermoid carcinoma of the anal canal. Eur J Surg Oncol. 2008;34:890-4.

24. Kroon BBK, Horenblas S, Deurloo EE, Nieweg OE, Teerstra HJ. Ultrasonography-guided fine-needle aspiration cytology before sentinel node biopsy in patients with penile carcinoma. BJU. 2004;95:517-21.

25. Van Rijk MC, Deurloo EE, Nieweg OE, Gilhuijs KG, Peterse JL, Rutgers EJ, et al. Ultrasonography and fine-needle aspiration cytology can spare breast cancer patients unnecessary sentinel lymph node biopsy. Ann Surg Oncol. 2006;13:31-5.

26. Van Rijk, MC, Teerstra HJ, Peterse JL, Nieweg OE, Olmos RA, Hoefnagel CA, et al. Ultrasonography and fine-needle aspiration cytology in the preoperative evaluation of melanoma patients eligible for sentinel node biopsy. Ann Surg Oncol. 2006;13: 1511-6.

27. Crawshaw JW, Hadway P, Hoffland D, Bassingham S, Corbishley CM, Smith Y, et al. Sentinel lymph node biopsy using dynamic lymphoscintigraphy combined with ultrasound-guided fine needle aspiration in penile carcinoma. Brit J Radiol. 2009;82: $41-8$.

28. Nguyen BT, Joon DL, Khoo V, Quong G, Chao M, Wada M, et al. Assessing the impact of FDP-PET in the management of anal cancer. Radiother Oncol. 2008;87:376-82. 